\title{
A new knotless technique for combined transscleral fixation of artificial iris, posterior chamber intraocular lens, and penetrating keratoplasty
}

\author{
Efdal Yoeruek ${ }^{1} \cdot$ Karl U. Bartz-Schmidt ${ }^{1}$
}

Received: 9 September 2017 / Revised: 1 July 2018 / Accepted: 19 July 2018 / Published online: 12 September 2018

(c) The Royal College of Ophthalmologists 2018

\begin{abstract}
Purpose To present a new surgical technique for treating corneal opacity and aniridia with aphakia and the results in a small consecutive case series.

Methods A three-piece acrylic intraocular lens (IOL) was attached to a customized silicone iris prosthesis and fixed with three $10-0$ polypropylene sutures in a knotless technique using Z-sutures after trephination of the recipient cornea. The medical records of all consecutive patients who had received a keratoplasty and an implantation of an artificial iris and IOL were reviewed.

Results Five eyes of five patients were included in the analysis. The mean age of the patients was 46.2 years and the mean follow-up was 24.6 months. The mean best-corrected visual acuity improved from $1.36 \operatorname{logMAR}$ before surgery to 0.78 $\log$ MAR after surgery during the follow-up. At the last follow-up visit, the artificial iris-IOL complex was well centered with good positioning in all cases.

Conclusions Management of post-traumatic aniridia combined with aphakia and corneal scars or graft failure by haptic fixation of a foldable IOL on an artificial iris combined with a simultaneous keratoplasty appears to be a promising approach, which allows to correct a complex lesion with a less traumatic and faster procedure.
\end{abstract}

\section{Introduction}

Due to severely damaged ophthalmic structures, cases with post-traumatic aniridia combined with aphakia and corneal opacity are highly challenging [1]. Often, multiple previous surgeries have been performed in these cases. Due to large iris defects, corneal opacities, and aphakia, the visual acuity is severely diminished and the depth of focus and contrast sensitivity are compromised [2]. For correction of large iris defects, an artificial iris (Human Optics AG) was developed for these cases. It has been commercially available since 2007 under the name of "Artificial Iris". This device is a three-layer sandwich with a central thin-fiber meshwork

Electronic supplementary material The online version of this article (https://doi.org/10.1038/s41433-018-0202-4) contains supplementary material, which is available to authorized users.

Efdal Yoeruek

Efdal.Yoeruek@googlemail.com

1 Department of Ophthalmology, Eberhard-Karls University, Tuebingen, Germany coated on both sides with silicone layers. The fiber layer enables the suturing of the implant. The anterior layer is colored like the natural iris by incorporating various medical-grade pigments into the silicone filler, allowing color matching with the remaining iris tissue in the same eye or the iris in the fellow eye. The posterior layer is colored uniformly black to completely block light transmission [3].

The key question is how to handle these challenging cases with minimal intraoperative and postoperative complications and how to achieve high patient satisfaction. We present a new surgical technique for treating these highly challenging patients in a single operation and report the outcomes of five patients.

\section{Materials and methods}

\section{Clinical data: patients and postoperative follow-up}

The medical records of all consecutive patients who had received a keratoplasty and an implantation of an artificial iris and IOL were reviewed. 

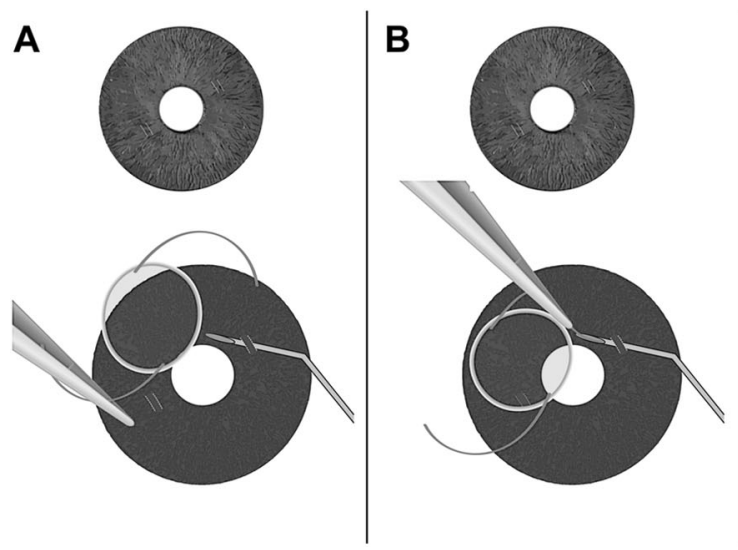

Fig. 1 Preparation of the artificial iris-IOL complex. a Two small stab incisions using a $0.9-\mathrm{mm}$ microsurgical blade are created about $1 \mathrm{~mm}$ apart, passing through the back of the customized iris prosthesis. b The haptics of a three-piece IOL are docked at the tip of a 27 -gauge hollow syringe and pulled through the small previously created tunnel in the

\section{Surgical technique}

The artificial IOL complex was prepared as described previously [4]. For each patient, the iris diaphragm was individually trephanized to a diameter of the white-to-white distance $-1 \mathrm{~mm}$. At three points, $120^{\circ}$ apart from each other, 10.0 polypropylene armed sutures were fixed $1 \mathrm{~mm}$ from the rim of the artificial iris (Fig. 1). The conjunctiva was opened circumferentially at the limbus, a Flieringa ring was sutured to the sclera, and an infusion cannula was positioned in the pars plana in the temporal inferior quadrant. When necessary, the remaining anterior vitreous was removed by vitrectomy after placing an additional cannula. At $1.2 \mathrm{~mm}$ from the limbus, three 27-gauge hypodermic needles were inserted before opening the eye globe. The cornea was trephanized with the Barron-Hessburg system. All three 10.0 polypropylene sutures were drawn through the preplaced needles and the prefabricated artificial iris complex was inserted into the eye globe. Following this, the prepared donor cornea was sutured with a double-running anti-torque suture. Using the pars plana infusion to keep the intraocular pressure controlled, the prosthesis was then centered and fixed to the sclera using a Z-suture technique [5]. The infusion cannula and the Flieringa ring were removed and the conjunctiva was closed (Video 1, Fig. 2).

\section{Results}

\section{Visual outcome}

Five eyes of the five patients were included in this analysis. The mean age of the patients was 46.2 years and the mean follow-up was 24.6 months. Table 1 shows the preoperative
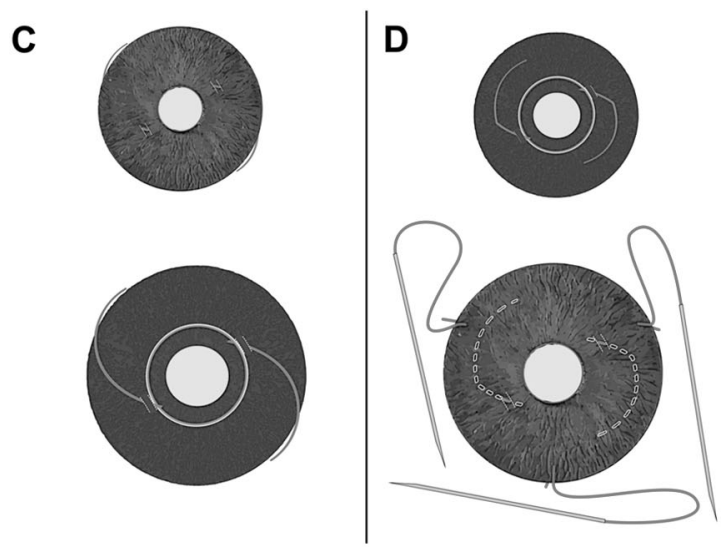

iris prosthesis. $\mathbf{c}$ Because of the high elasticity and stability of the iris prosthesis, the haptics are tightly attached to the back of the artificial iris. d Three 10.0-armed polypropylene sutures were fixed at the 12, 4, and 8 o'clock positions

characteristics and the follow-up of each patient. All five patients had received a combined penetrating keratoplasty and transscleral fixation of an artificial iris and IOL complex. Penetrating keratoplasty was performed because of corneal or graft injury secondary to trauma. Anterior vitrectomy was performed at the time of surgery in two cases (40\%). The mean best-corrected visual acuity improved from $1.36 \log$ MAR before surgery to $0.78 \log$ MAR after surgery during the follow-up. Data on glare and photophobia sensation were available for three patients. In two patients, best-corrected visual acuity was too low, so that no changes could be achieved. In three cases $(60 \%)$ glare sensation was reduced. Postoperative complications included one graft failure (20\%) which occurred during the first year after surgery. In this case, uncontrolled episodes of high intraocular pressure were noted. Moreover, vascularization in two quadrants of the cornea was registered preoperatively, so that a graft rejection cannot be ruled out. The systemic steroid application was increased in this case. However graft failure could not be prevented. Glaucoma was present in three patients $(60 \%)$ preoperatively. In two cases the intraocular pressure could be sufficiently controlled postoperatively. No patient developed new-onset glaucoma after surgery during the follow-up. At the last follow-up visit, the artificial iris-IOL complex was well centered with good positioning in all cases (Fig. 3). There were no cases of retinal detachment. None of the cases developed macular edema during the follow-up.

\section{Discussion}

In traumatic aniridia, the eye's anterior segment, including the cornea, is often severely damaged. Serious 

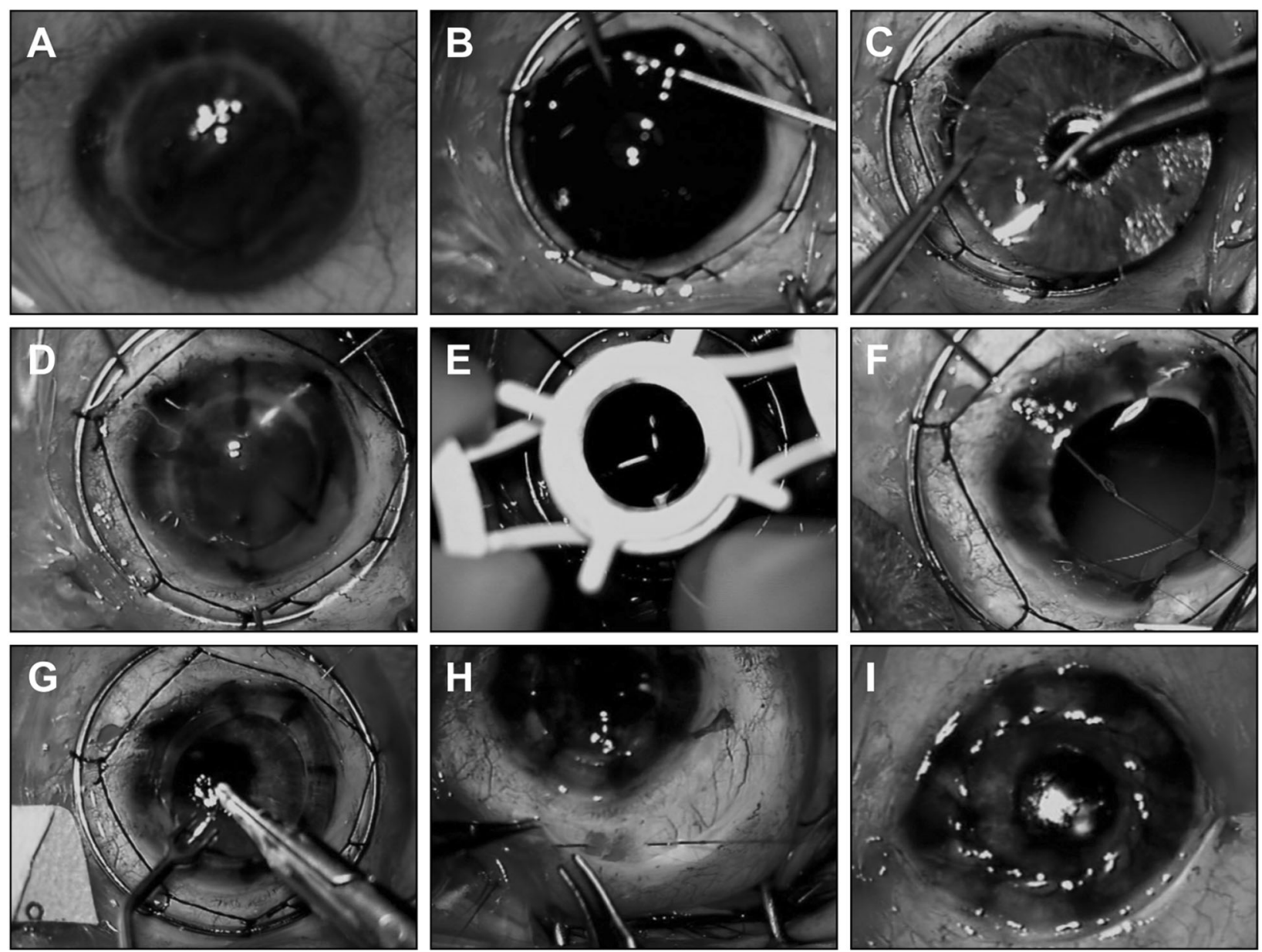

Fig. 2 Intraoperative images. a Before surgery. b Fixation of the threepiece IOL onto the artificial iris via the haptics. c Knotting the 10.0 polypropylene sutures to the artificial iris-IOL complex. d Insertion of three 27 -gauge needles at the corresponding areas at $1.2-\mathrm{mm}$ distances to the limbus. e Opening the eye globe. f Pulling the sutures through the needles. g Suturing the corneal graft. h Fixation of the artificial iris-IOL complex to the sclera using Z-suture technique. i Closing the conjunctiva complications of iris loss often include corneal opacity, secondary glaucoma, retinal detachment, and cystoid macular edema [2]. Ocular trauma, including previous surgical trauma, was the cause of aniridia in all patients in our study. The loss of iris tissue explains complaints like glare and photophobia. Several procedures have been proposed to deal with this issue. Apart from intraocular surgical procedures to reduce these symptoms, corneal tattooing (keratopigmentation) has been proposed. We decided to use an iris diaphragm (artificial iris) in these patients. All patients required corneal transplantation combined with the artificial iris-IOL complex implantation.

We observed an increase of the best-corrected visual acuity despite the fact that three of five patients had ocular comorbidities which affected visual acuity. A graft failure was observed in one case due to an assumed graft rejection combined with an intraocular pressure elevation. Preoperatively, corneal vascularization was registered and the high-risk situation was discussed with the patient in great detail.

Traumatic aniridia with aphakia and corneal opacity demands extensive and complex reconstructive surgery. The extent and severity of the intervention can cause intraoperative and postoperative complications. No intraoperative complications were seen with the described technique in the cases presented.

Pre-existing secondary glaucoma is a common problem in traumatized eyes, especially in patients with traumatic aniridia [6]. In our series it was present in $60 \%$ of our patients. In addition, penetrating keratoplasty is a known risk factor for the development of glaucoma [7]. Preoperatively the intraocular pressure should be sufficiently controlled. With the surgical procedure used in our patients, the intraocular pressure was normal in all five cases for at least 6 months. Another common problem in these severely damaged eyes is the development of cystoid macular edema, especially after surgery. In none of our patients this 


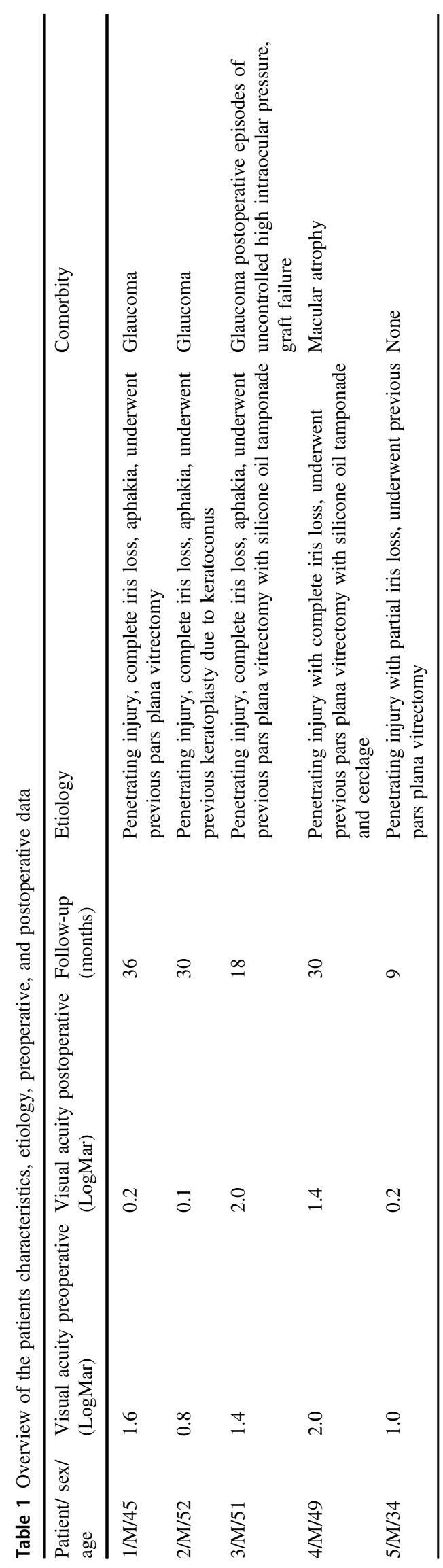

occurred. We believe that this can be attributed to our modified technique of individually adjusting the diameter of the iris diaphragm ( $1 \mathrm{~mm}$ smaller than the white-to-white distance). This helps to reduce irritation of the uveal tissue. We highly recommend this approach.

The novelty and the key step of our technique is the insertion of the 27 -g hypodermic needles before opening the eye globe. Moreover, the three-point $Z$-suture fixation technique offers the advantages of a knotless approach. Although difficulties with centration of other types of iris prosthesis have been described in the literature, the artificial iris complex remained well centered in all eyes in our series [8]. Thus, the three-point fixation allows a wellcentered artificial iris-IOL complex and seems to be sufficient.

To summarize, the management of post-traumatic aniridia combined with aphakia and corneal scars or graft failure by way of haptic fixation of a foldable IOL on an artificial iris simultaneously performed with keratoplasty appears to be a promising approach. It gives the surgeon the possibility to correct a complex lesion in one procedure, which means that it is both less traumatic and faster. The presented technique seems to be helpful in treating these challenging patients. Moreover, the custom-tailored iris prosthesis gives a perfect aesthetic result.

\section{Summary}

\section{What was known before}

- The treatment of post-traumatic aniridia combined with aphakia and corneal opacity is highly challenging. Due to a large iris defect, corneal opacity, and aphakia, the visual acuity is severely diminished and the depth of focus and contrast sensitivity are compromised.

- Several techniques and several iris implants have been described, combining penetrating keratoplasty, iris reconstruction, and IOL implantation.

\section{What this study adds}

- Corneal surgery combined with artificial iris (Human optics AG) and IOL implantation allows functional and esthetic rehabilitation in cases of corneal scars, major iris defects, and aphakia.

- The novelty and the key step of the presented technique is the insertion of the 27-g needles before opening the eye globe. Moreover, the three-point $\mathrm{Z}$-suture fixation technique offers the advantages of a knotless approach. By avoiding suture knots, the risk for scleral atrophy and suture erosion may be reduced. 
Fig. 3 Clinical photographs of patient 2. a Before surgery, complete iris loss, aphakia, and graft failure is observed. $\mathbf{b}$ At day 1 post-op. c 6 months postoperatively. d At the last visit, 30 months postoperatively. The artificial iris-IOL complex is well centered. The corneal graft is clear with no signs of failure during the whole follow-up
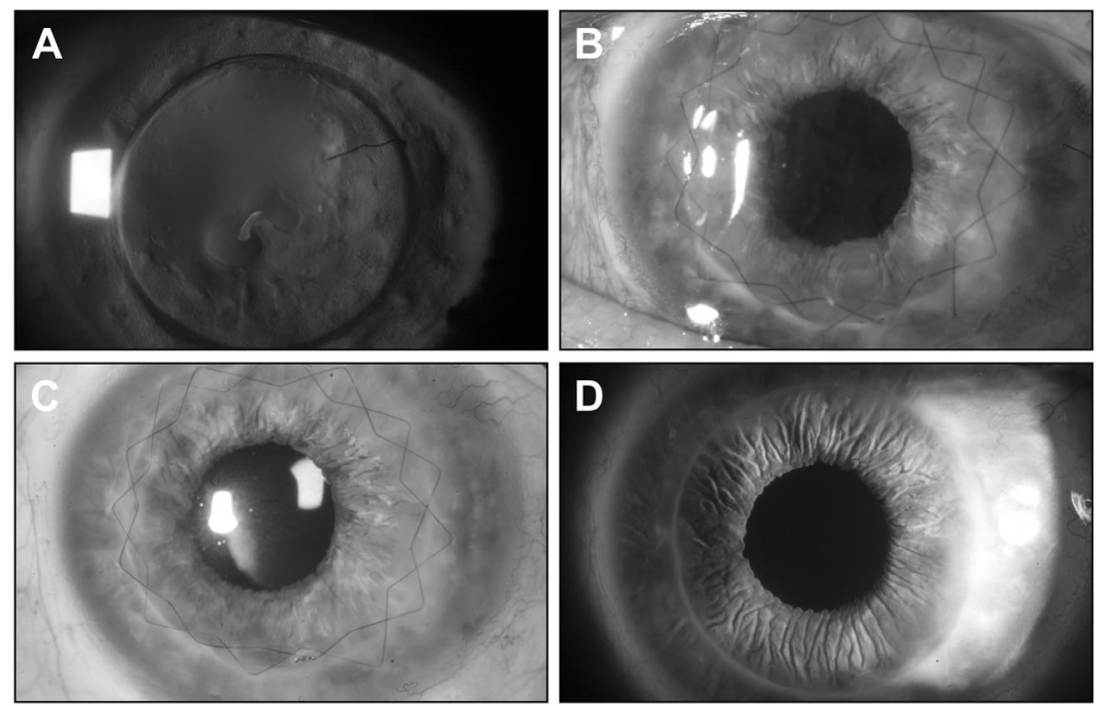

\section{References}

1. Aslam SA, Wong SC, Ficker LA, MacLaren RE. Implantation of the black diaphragm intraocular lens in congenital and traumatic aniridia. Ophthalmology. 2008;115:1705-12.

2. Thompson CG, Fawzy K, Bryce IG, Noble BA. Implantation of a black diaphragm intraocular lens for traumatic aniridia. J Cataract Refract Surg. 1999;25:808-13.

3. Koch KR, Heindl LM, Cursiefen C, Koch HR. Artificial iris devices: benefits, limitations, and management of complications. J Cataract Refract Surg. 2014;40:376-82.

4. Spitzer MS, Yoeruek E, Leitritz MA, Szurman P, Bartz-Schmidt KU. A new technique for treating posttraumatic aniridia with aphakia: first results of haptic fixation of a foldable intraocular lens on a foldable and custom-tailored iris prosthesis. Arch Ophthalmol. 2012;130:771-5.
5. Szurman P, Petermeier K, Aisenbrey S, Spitzer MS, Jaissle GB. Z-suture: a new knotless technique for transscleral suture fixation of intraocular implants. Br J Ophthalmol. 2010;94:167-9.

6. Bojikian KD, Stein AL, Slabaugh MA, Chen PP. Incidence and risk factors for traumatic intraocular pressure elevation and traumatic glaucoma after open-globe injury. Eye. 2015;29: 1579-84.

7. Chien AM, Schmidt CM, Cohen EJ, Rajpal RK, Sperber LT, Rapuano CJ, et al. Glaucoma in the immediate postoperative period after penetrating keratoplasty. Am J Ophthalmol. 1993; 115:711-4.

8. Mashor RS, Bahar I, Kaiserman I, Berg AL, Slomovic A, Rootman DS. Combined penetrating keratoplasty and implantation of iris prosthesis intraocular lenses after ocular trauma. J Cataract Refract Surg. 2011;37:582-7. 